\title{
The Effect of Fulvic Acid on the Dose Effect of Selenite on the Growth of Wheat
}

\author{
A. Peng, ${ }^{*}$ Y. Xu, AND Z. J. Wang \\ SKLEAC, Research Center for Eco-environmental Sciences, \\ Chinese Academy of Sciences, Beijing 100085, \\ People's Republic of China \\ Received February 21, 2001; Revised April 10, 2001; \\ Accepted May 21, 2001
}

\begin{abstract}
The effect of fulvic acid on the dose effect of selenite has been studied by using the hydroponics culture of wheat in the presence of selenite and fulvic acid (FA). The bioavailability of Se was investigated by 5 morphological end points and 12 biological and biochemical end points at different phases of growth of wheat seedling and seed germination. The "platform" of the dose effect observed indicates that the presence of FA can have both the beneficial effects and antagonists on the toxicity of Se to a certain extent.
\end{abstract}

Index Entries: Selenium; fulvic acid; dose-effect relationship; wheat; hydroponic culture.

\section{INTRODUCTION}

In the soil-biological ecosystem, plants uptake and accumulate Se from soil and bring it into the food chain. Plants, especially corn, play an important role in the nutritional and harmful effects of se for human and animal. There are high-selenium regions in China, in which the content of Se in corn is hundreds of times that in normal areas, with the result that selenosis for human and animal has been found (1). In addition, Se compounds added to cropland has been used to raise the content of selenium in the grain of crops for controlling the endemic diseases such as Kechan

*Author to whom all correspondence and reprint requests should be addressed. 
disease and Kashin-Bck disease in China $(2,3)$. The chemical speciation of Se in soil is important for its uptake and accumulation. The interaction between Se and soil humic substances could be one of the top considerations regarding Se speciation.

Humic acid is the major component of soil organic matter and takes about $85-90 \%$ of the soil organic carbon. It was reported that the chemical form of Se in soil was mainly a humat-bound form $(4,5)$. The influences of fulvic acid (water-soluble fraction of humic substances, FA) on the bioavailability and toxicity of selenite for cells (6) and animals (7) has been studied. It was found that antagonism existed between FA and selenite. Our group has previously studied the effects of FA on the bioavailability and toxicity of selenite for wheat $(8,9)$. In the presence of FA, the uptake of Se strongly decreased by wheat seedling (10). When the concentration of Se was low, the germination rate of the wheat seed was accelerated by FA (11). The dose-effect relationship for Se on the growth of wheat has been reported by measuring 5 end points in morphological studies and 12 end points in biological and biochemical studies during different phases of growth of the wheat seedling and germination in a hydroponic culture (12).

In this article, previous data are summarized and the dose-effect relationship of Se in the presence of FA is studied. It was found that the doseeffect relationship of Se in the presence of FA is much wider than that without FA. It is suggested that FA could affect the beneficial and toxic effects of Se to a certain extent and the function of FA on the Se behavior has been discussed from the data.

\section{MATERIAL AND METHOD}

\section{Incubation}

Wheat seeds (Triticum aestivum L., H-65) were germinated in a culture bottle at $25^{\circ} \mathrm{C}$ for $3 \mathrm{~d}$. The seedling were transferred to Hoagland's solution containing, if added, various concentration of sodium selenite and fulvic acid during the seed germination or seedling growth period. The experimental container was illumined at $3500 \mathrm{Lx}$ and a temperature of $200^{\circ} \mathrm{C}$. According to the test, request samples were taken in various times.

The fulvic acid was extracted from drab soil using $0.1 \mathrm{~mol} \mathrm{NaOH}$ and was purified using the method recommended by the International Committee of Humic Acid (13).

\section{Method and Data Treatment}

The assay methods are the same as described in our previous report (10). 


\section{RESULTS AND DISCUSSION}

The morphological changes and the biological and biochemical indices of wheat in a hydroponic culture containing selenite during the seed germination or seedling growth period have been measured with or without FA. In the presence of FA $(50 \mathrm{mg} / \mathrm{L})$, stimulation effects were observed when the Se concentration was low (0.1-1.0 mg/L). For example, the germination rate in the Se-FA group was significantly higher than that in the Se group with a lower dosage. The root length in the Se-FA group was found to be longer than that in the Se group when the selenite concentration was below $0.5 \mathrm{mg} / \mathrm{L}$. The enzymatic activity of $\alpha$ amylase in the Se-FA group is found to be higher than that in the Se group. Especially, the toxicity of selenite can be antagonized by FA. In the Se group with greater than $5.0 \mathrm{mg} / \mathrm{L}$ selenite, the wheat seedling was found to be shorter, the stem thin, and the leaf yellowish at the top; it was shrunken by the lack of water supply and the morphological alteration was more serious in the $10-\mathrm{mg} / \mathrm{L}$ treatment. In the presence of FA, most of the toxic symptoms had not been observed. Furthermore, in the absence of FA, the inhibition effect of selenite for the synthesis of chlorophyll is $>1 \mathrm{mg} / \mathrm{L}$, and in presence of FA, it is $>6 \mathrm{mg} / \mathrm{L}$. The harmful effect of selenite on wheat growth results were found in the increase of cell membrane permeability and free-proline content, whereas in the presence of FA, both phenomena could be reduced. It was shown that the presence of FA limits Se uptake through reducing the bioavailablity of Se by the plant root when the concentration of selenite was higher than $1 \mathrm{mg} / \mathrm{L}$.

The critical threshold of selenite with and without FA of the six end points for morphology and the 15 end points for physiology and biochemistry with different phases of growth of the wheat seedling and seed germination are summarized in Table 1.

The scheme of the dose-biological effect relationship of both Se and Se-FA groups are shown in Fig.1. From this figure, it can be seen that the "plateau" of the curve of Se-FA group is found to be much wider than that without FA, indicating that FA coaffects the beneficial effects and antagonists of the toxicity of selenite to a certain extent. It indicated that the region of best nutrition concentration of selenite increases in the presence of FA. In addition, the toxic effects of Se drop strongly at $>5 \mathrm{mg} / \mathrm{L}$. In the presence of FA when Se(IV) is approx $5 \mathrm{mg} / \mathrm{L}$, the wheat growth was not inhibited. Both Se and FA coaffect under the situation of a "plateau"; for example, the germination rate was induced, seedling chrolophyll and protein content increased, and the promotion of fresh weight as well as height were more than that in the presence of selenium or FA only. It was suggested that in the high-Se region, the toxic effect would be reduced if the soil contains a higher concentration of FA. 
Table 1

The Critical Threshold of Selenite Content in the Se-FA Group in Wheat

\begin{tabular}{c|cccc}
\hline \multirow{2}{*}{ Items } & \multicolumn{4}{|c}{ Threshold for Se (mg/L) } \\
\cline { 2 - 4 } Germination rate & Beneficial dose & Optimum dose & Adverse dose & Toxic dose \\
\cline { 2 - 4 } Seed vigor & $\sim 5.0(\sim 1.0)^{*}$ & $0.1-1.0(0.5)$ & $>5.0(>1.0)$ & $40.0(20.0)$ \\
Bud length & $\sim 10.0(-1.0)$ & $0.1-0.5(0.1)$ & $>10.0(>1.0)$ & $20.0(5.0)$ \\
Seedling fresh weight & $\sim 5.0(\sim 1.0)$ & $0.1-1.0(>1.0)$ & $>5.0(>1.0)$ & $10.0(10.0)$ \\
Seedling height & $\sim 5.0(\sim 1.0)$ & $0.1(>0.1)$ & $>5.0(>1.0)$ & $10.0(5.0)$ \\
Membrane permeability & $\sim 5.0(\sim 1.0)$ & $0.1(>0.1)$ & $>5.0(>1.0)$ & $>10.0(10.0)$ \\
Free proline & $\sim 5.0(\sim 1.0)$ & $0.1(>0.1)$ & $* *(>1.0)$ & $* *(10.0)$ \\
Chlorophyll A & $\sim 5.0(\sim 1.0)$ & $0.5(>0.1$ & $>5.0(>1.0$ & $* *(5.0)$ \\
Chlorophyll B & $\sim 5.0(1.0)$ & $0.1(>0.1)$ & $>5.0(>1.0)$ & $10.0(10.0)$ \\
Chlorophyll A+B & $\sim 5.0(\sim 1.0)$ & $0.1(>0.1)$ & $>5.0(>1.0)$ & $10.0(10.0)$ \\
Chlorophyll K & $\sim 5.0(\sim 1.0)$ & $0.1(>0.1)$ & $>5.0(>0.1)$ & $10.0(5.0)$ \\
Sugar content & $<5.0(<0.5)$ & $0.1(>0.5)$ & $>5.0(>1.0)$ & $10.0(5.0)$ \\
Activity a-amylase & $\sim 5.0(\sim 1.0)$ & $0.1-0.5(>1.0)$ & $>5.0(>1.0)$ & $20.0(20.0)$ \\
Activity B-amylase & $\sim 5.0(\sim 1.0)$ & $0.1)>1.0)$ & $>5.0(>1.0)$ & $10.0(10.0)$ \\
Protein content & $* *(* *)$ & $1.0(* *)$ & $* *(* *)$ & $* *(* *)$ \\
Malonic dialdehyde & $\sim 1.0(1.0)$ & $0.1(>1.0)$ & $>5.0(>1.0)$ & $10.0(5.0)$ \\
Activity of POD & $<5.0(\sim 1.0)$ & $0.5(<1.0)$ & $>5.0(>1.0)$ & $20.0(10.0)$ \\
\hline
\end{tabular}

The data in the parentheses are the threshold of Se without FA.

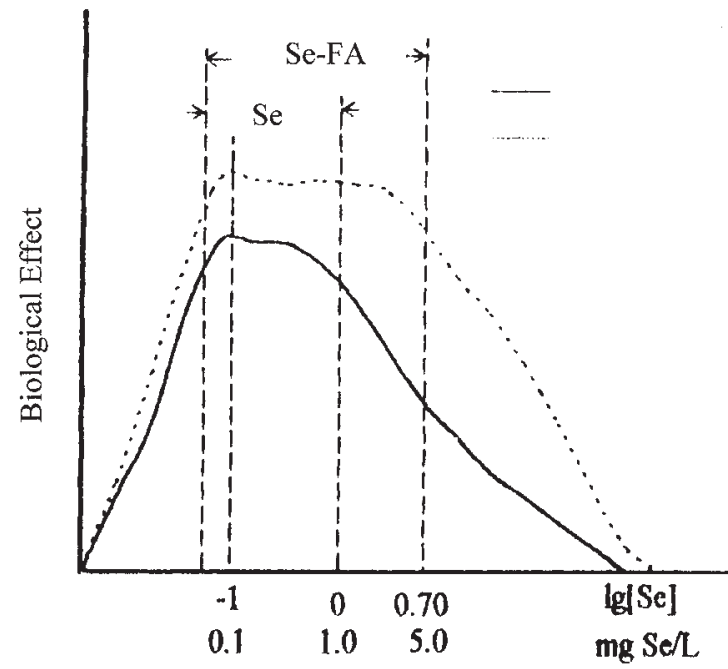

Se concentration

Fig. 1. The plot of the dose-effect relationship of Se (-) and Se-FA (- - -) groups. 


\section{REFERENCES}

1. D. Mao, H. Su, L. Yan, S. Wang, and X. Yu, Epidemic inverstigation of endemic selenium poisoning in the west of Hubai Provence in China, Chin. J. Endemiol. 9(5), 311-314 (1990).

2. J. Xu, Observation of the effect of treatment of Keshan-Disease by universal Se supplementation, Chin. J. Endemiol. 13(1), 31-33 (1994).

3. D. Chen, S. Ren, X. Wang, and J. Li, The prevention and treatment effects on Kaschin-Beck Disease of application selenium fertilizer, in Environmental Life Elements and Health, J.A. Tan, P.J. Peterson, R.B. Li, and W.Y. Wang, eds., Science Press: Beijing, pp. 352-355 (1990).

4. Z. Wang, B. Peng, and A. Peng, The effect of organic matters in water and soil from Kachin-Beck Disease region on the speciation and bioavailability of selenium, J. Environ. Sci. (China) 12(4), 86-90 (1991).

5. M. M. Abrams, R. G. Burau, and R. J. Zasoski, Organic selenium distribution in selected California soils, Soil Sci. Soc. Am. J., 54, 979-982 (1990).

6. A. Peng and C.L. Yang, Examination of the role of selenium in the Kashin-Beck Disease. Cartilage cell test and model studies. Biol. Trace Element Res. 28(1), 1-10 (1991).

7. A. Peng and L. Xu, Antagonism between selenium and humic acid, Sci. Sin. (Series B) 30(1), 66-74 (1987).

8. Y. Xu, Z. Wang, W. Wang, and A. Peng, Effect of selenium and fulvic acid on seed germination of wheat and its physiological properties, Chin. J. Appl. Ecol. 8(4), 439-444 (1997).

9. A. Peng, Y. Xu, and Z. J. Wang, Study on the incorporation of selenium into eroxidase isozyme of wheat seedling, Biol. Trace Element Res. 70, 117-125 (1999).

10. Y. Xu, Z. J. Wang, and A. Peng, Effect of fulvic acid in environment on the uptake selenium of wheat, J. Ecol. (China) 16, 135-139 (1996).

11. Z. J. Wang, Y. Xu, and A Peng, Influences of fulvic acid on bioavailability and toxicity of selenite for wheat seedling and growth, Biol. Trace Element Res. 55, 147-162 (1996).

12. A. Peng, Y. Xu, J. H. Liu, and Z. J. Wang, Study on the dose-effect relationship for selenite on the growth of wheat, Biol, Trace Element Res. 26(2), 175-181 (2000).

13. A. Peng and W. Hua, Humic acid in water and its complexes-extraction of humic acid from water and soil, ACTA Sci. Circum. 1, 126-131 (1981). 